\title{
AN EFFICIENT SPECTRUM SHARING METHOD BASED ON GENETIC ALGORITHM IN HETEROGENEOUS WIRELESS NETWORK
}

\author{
Kazuhiko Kinoshita $^{1}$, Masashi Nakagawa ${ }^{1}$, Keita Kawano ${ }^{2}$, and Koso \\ Murakami $^{1}$ \\ ${ }^{1}$ Graduate School of Information Science and Technology, Osaka University, Japan \\ ${ }^{2}$ Information Technology Center, Okayama University, Japan
}

\begin{abstract}
With advances in wireless communication technologies, users can have rich contents not only via wired networks but also via wireless networks such as Cellular, WiFi, and WiMAX. On the other hand, however, lack of spectrum resources becomes an important problem for future wireless networks. To overcome this problem, dynamic spectrum access technology receives much attention. In this paper, we propose a novel spectrum sharing method based on genetic algorithm in which a WiFi system temporarily uses a spectrum band of WiMAX system in WiFi/WiMAX integrated networks as a typical heterogeneous wireless network. Finally, we confirm the effectiveness of the proposed method by simulation experiments.
\end{abstract}

\section{KEYWORDS}

Heterogeneous Wireless Network, Dynamic Spectrum Access, Spectrum Sharing, GA

\section{INTRODUCTION}

With advances in communication technologies, network services provided via the Internet have been widely diversified. Users can have such services not only via wired networks but also via wireless networks. In wireless networks, the bandwidth of cellular networks is growing constantly, and the number of wireless LAN access points (APs) is increasing greatly, as the demand for multimedia services is growing as much as in wired networks [1].

On the other hand, there are several wireless systems in practical use such as Cellular [2], WiFi [3], and WiMAX [4,5]. Each system uses its own spectrum as prescribed by law to avoid radio interference.

In addition, the wireless communication systems described above are used independently, because the mechanisms of these systems are fundamentally different. Thus, a user must switch between systems manually. To overcome such an inconvenient situation, the integrated network [6,7], in which these systems interwork, has been designed for the next generation wireless communication system. In such an integrated network, mobile users can have seamless, continual communication via the best wireless communication system, according to the applications or the conditions of wireless systems. Therefore, it is possible to provide better communications for mobile users [8].

DOI : $10.5121 /$ ijenc. 2014.6510 
However, available spectrum resources are finite, so that another approach that uses radio resources more effectively is desirable. As mentioned above, although the amount of available radio spectrum for particular wireless communications is decreasing because of the diversity of wireless networks, the traffic demand for wireless networks increases with the growth of various broadband applications. To overcome this dilemma, Cognitive Radio $[9,10]$ receives much attention.

The cognitive radio technology can be classified into multi-mode system and dynamic spectrum access (DSA) [11,12]. Multi-mode system selectively avails of a number of wireless systems according to the communication environment of users and the condition of each wireless system. On the other hand, in DSA, a wireless system can secondarily avail of the radio frequency spectrum that other wireless systems are using [13]. Frequency spectrum is used more efficient by DSA than by multi-mode system.

The authors proposed the method that shares the spectrum between WiMAX and WiFi by using DSA to reduce call blocking probability for streaming services in WiMAX/WiFi integrated network [14]. However, wireless communication traffic includes not only QoS traffic such as multimedia streaming but also BE traffic such as file downloading. Thus, in this paper, we propose a spectrum sharing method to improve throughput by using DSA in WiMAX/WiFi integrated network. To find a better spectrum assignment pattern for throughput improvement, we use Genetic Algorithm (GA) $[15,16]$ which can detect multiple solution in parallel.

The rest of this paper is organized as follows. Section 2 introduces some related works and points out the problematic issues. Section 3 proposes the novel spectrum sharing method and it is evaluated by simulation experiments in Section 4. Finally, Section 5 makes some conclusions and show future work.

\section{RELATED WORK}

\subsection{Heterogeneous Wireless Network}

Although several wireless systems, such as Cellular, WiFi, and WiMAX, have developed independently, they should be integrated for seamless access from users. Therefore, in recent years, the Cellular/WiFi integrated network [17] and WiMAX/WiFi integrated network [18] have been researched actively.

Especially, WiMAX/WiFi integrated network achieves high-quality communication by using WiMAX and WiFi as complementary access resources. This integrated network enables load balancing between WiMAX and WiFi by using each system selectively in response to the demands of users and the condition of each system.

However, this integrated network assumes that each wireless system uses the spectrum band prescribed by law, so that even if the WiMAX system has unused spectrum temporarily, it cannot be used by WiFi systems. To overcome this problem, cognitive radio receives much attention.

\subsection{Dynamic Spectrum Access}

According to [12], DSA strategies can be categorized into three models; Dynamic Exclusive Use Model, Open Sharing Model, and Hierarchical Access Model. 
Dynamic exclusive use model protects the current spectrum regulation policy, which spectrum bands are licensed to services for exclusive use. Open sharing model openly shares among peer users as the basis for managing a spectral region. Hierarchical access model adopts a hierarchical access structure with primary (licenses) and secondary users.

\subsection{Existing Method in WiMAX/WiFi Integrated Network and Problematic Issues}

A spectrum sharing method that several WiFi APs temporarily uses an unused spectrum band of WiMAX in WiMAX/WiFi integrated network is proposed [12]. It is based on the spectrum overlay described in 2.2.3. In this proposal, as shown in Fig. 1, a central control server named spectrum manager (SM) manages the spectrum assignment and necessary information for assignment in a WiMAX base station (BS) and WiFi APs inside the WiMAX service area of the BS. In this paper, we call the WiMAX service area "area" and the hexagonal area that can be deposited WiFi AP "cell".

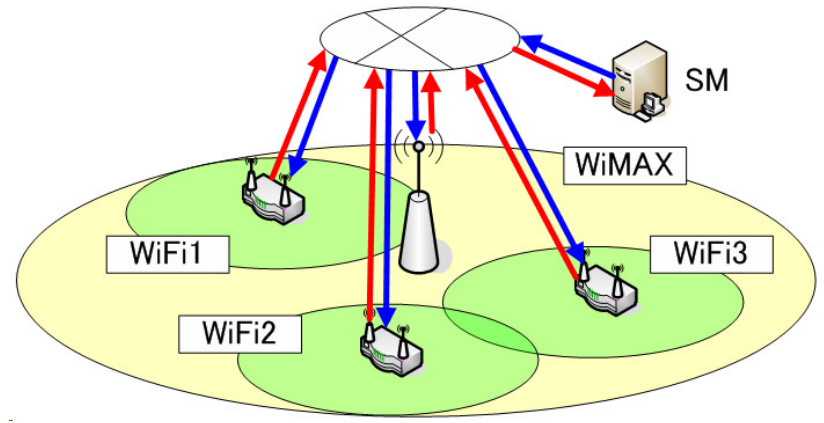

Figure 1. Network model

The coverage area of WiMAX BS, about a few kilometers in radius, is so wide that it includes some WiFi APs. Therefore, the same spectrum can be repeatedly used by assigning unused spectrum of WiMAX to WiFi AP without causing interference between the adjacent WiFi APs. If two or more WiFi APs use a spectrum of the WiMAX BS, the spectrum utilization efficiency can be enhanced for the whole network.

[13] proposed the method that classified cells in whole network into three parties of A, B, C as shown in Fig. 2. APs that belong to the same party can use the same spectrum without radio interference. Therefore, all WiFi APs are classified into three parties and the proposed method assigns a spectrum to one of them.

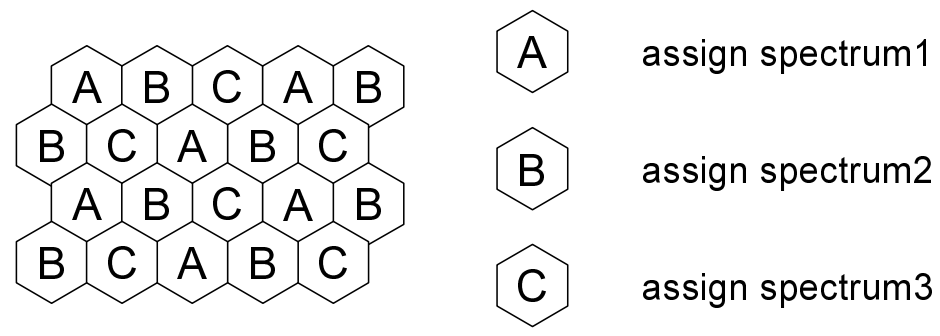

Figure 2. Assignment of spectrum to three parties

This method sums up the estimated number of blocking calls for every party and finds the party with the maximum number. Then, this method attempts to assign a spectrum from WiMAX BS to WiFi APs belonging to the party and reevaluates the total number of blocking calls for the whole network in a certain period time with this configuration. Finally, this method compares 
the total number of blocking calls for the whole network before the spectrum assignment and after. If the latter is smaller, this method carries out the spectrum assignment and repeats these procedures. It is confirmed that this method achieves effective use of spectrum adaptively.

This method uses just three spectrum assignment patterns, however, so that it may miss a better spectrum assignment pattern. In addition, the aim of this method is only to reduce the call blocking probability of QoS communications. Therefore, it does not consider the throughput of BE services.

\section{Spectrum Sharing baSEd On Genetic Algorithm}

\subsection{Overview}

To overcome the problem described in previous section, we propose a method to find WiFi APs to be assigned an additional spectrum based on GA. Here, we use the sum of the load of WiFi AP as a fitness value and disallow to assign the same spectrum to adjacent WiFi APs at the same time as a constraint.

Specifically, we assume $n$ cells in an access area of a WiMAX BS as shown in Fig. 3, where each number is treated as a cell ID. We prepare a spectrum assignment list to WiFi AP as shown in Table 1. The index of this list corresponds to cell ID, and the element of the list "1" means that the cell can be an assignment target. We apply this list as an individual in GA.

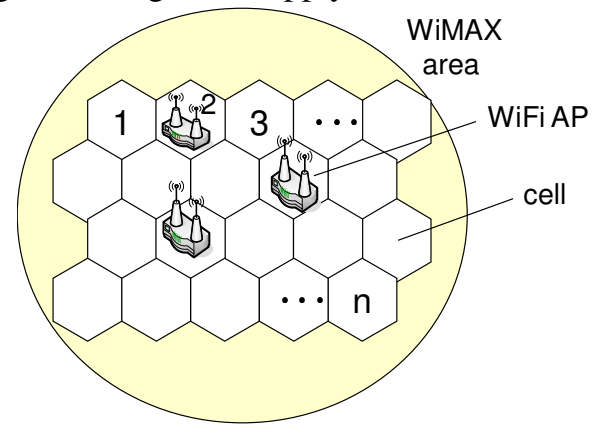

Figure 3. Cell ID

Table 1. Spectrum assignment list

\begin{tabular}{|c|c|c|c|c|c|}
\hline ID & 1 & 2 & 3 & $\cdots$ & $n$ \\
\hline element & 0 & 1 & 0 & $\cdots$ & 1 \\
\hline
\end{tabular}

Fig. 4 shows the flowchart of the proposed method. First, the proposed method generates $l$ individuals and define the set of them as an initial group. Next, it calculates fitness value of each individual and keeps the individual that has the highest fitness value as a candidate individual. Then, it makes a crossover for a pair of individuals and calculates the fitness value of each reproduced individual. If this value is higher than that of the candidate individual, this individual is kept as a new candidate individual. Next, it executes a selection process to keep the number of individuals as many as before a crossover. On the other hand, a mutation process is invoked to seek for variety of patterns when all individuals are identical. 


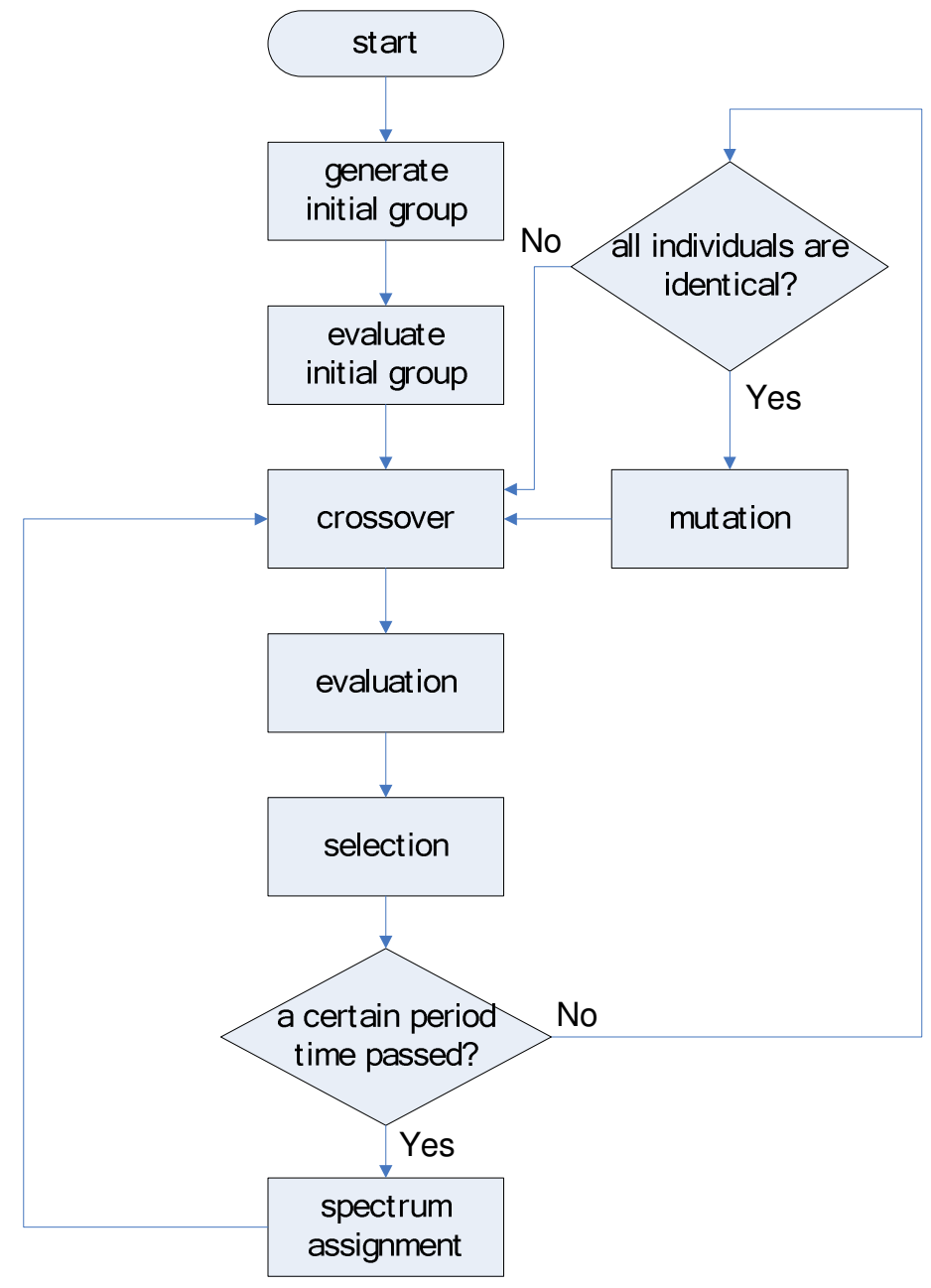

Figure 4. Flowchart of the proposed method

After repeating the above operation in a certain period of time, the proposed method decides to assign a spectrum to one or more $\mathrm{WiFi} \mathrm{AP}(\mathrm{s})$ according to the candidate individual with the highest fitness value. Note here that the proposed method does not stop after a certain times of iteration but keeps to seek for better individuals.

In the following subsections, we explain the procedures of the proposed method in detail.

\subsection{Initialization}

First, the proposed method randomly generates $l$ individuals and we define the set of them as an initial group. Specifically, the proposed method randomly selects a cell $i$ which has WiFi AP whose adjacent cells have the element " 0 " to be allocated the spectrum. This operation is repeated till WiFi AP which can be allocated spectrum does not exist, and we define the pattern after above iteration as individual. This procedure of individual generation is repeated until the number of individuals is $l$. 


\subsection{Crossover}

We introduce a cluster as a set of adjacent seven cells such as 1-7, 8-14, 15-21, and 22-28 in Fig. 5. The proposed method makes a crossover by this cluster. The cluster which has the same ID for each individual consists of the same cells, so that any cells without WiFi AP are never target of spectrum assignment.

First, the proposed method randomly selects two individuals $\alpha$ and $\beta$ as parents from $l$ individuals, and also randomly selects $k$ clusters in each individual. After that, the proposed method exchanges the elements between $\alpha$ and $\beta$, and repeats this process for $m$ pairs of parents.

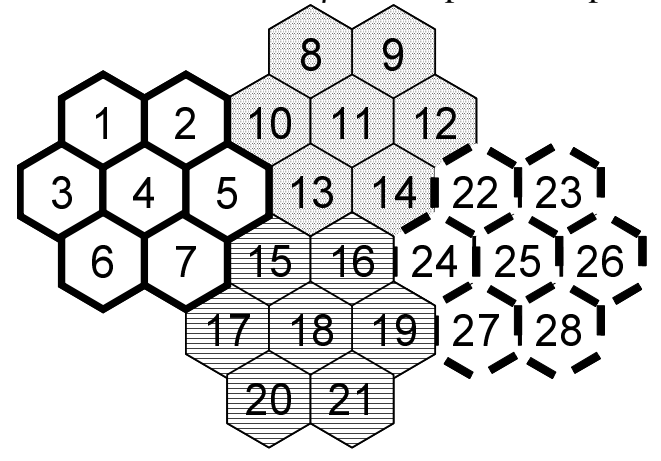

Figure 5. Example of cluster

Fig. 6 shows the execution example of a crossover. The value shown in each cell represents the element of the spectrum assignment list. Suppose to make a crossover by the thick boxed clusters in $\alpha$ and $\beta$. The element of the list corresponding to cell ID of cluster between $\alpha$ and $\beta$ is exchanged, and new spectrum assignment lists, $\alpha^{\prime}$ and $\beta^{\prime}$ as children, are generated. Then these children are added to the initial group as new individuals. Because $\alpha$ and $\beta$ are belonging to the initial group, as a result, the number of individuals increases by two.
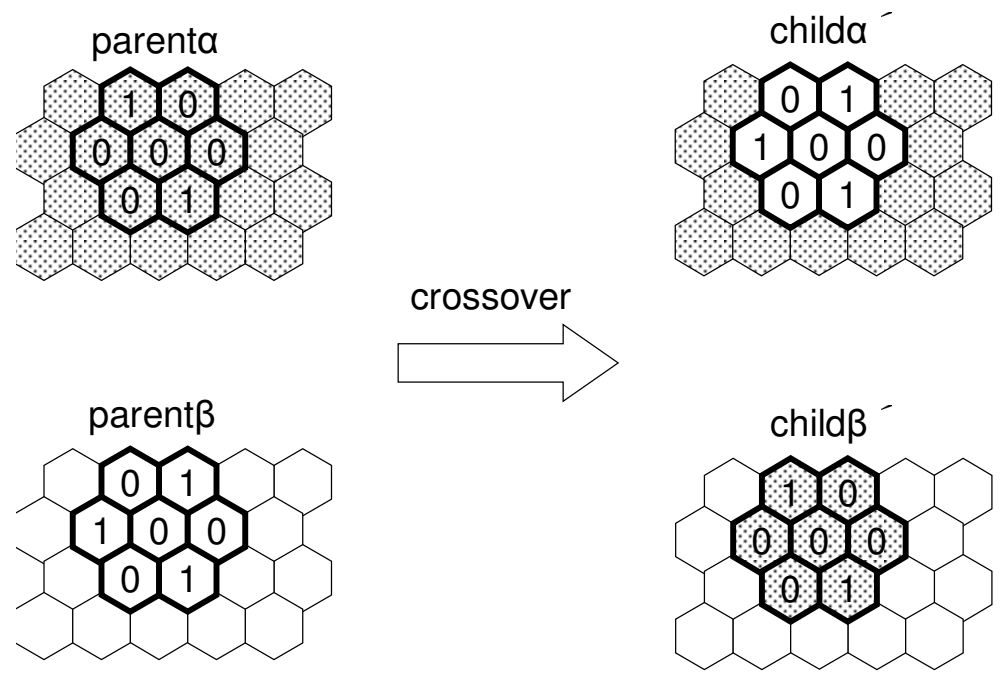

Figure 6. Example of crossover by cluster

However, a child generated by a crossover may cause radio interference. Therefore, in such a case, to satisfy the constraint, the proposed method excludes the WiFi AP which has the lowest impact on the fitness value from assignment candidates. We explain this process with Figs. 7, 8 
and 9. Suppose that the assignment pattern of $\alpha$ ' in Fig. 6 is as shown in Fig. 7 specifically and interference occurs in the cell circled by solid line. In the case where the load of each AP is as shown in Fig. 8, the element of the cell where the load is 15 changes to " 0 ". On the other hand, if the load of each AP is as shown in Fig. 9, the element of the cells where the load are 7 and 8 changes to " 0 ".

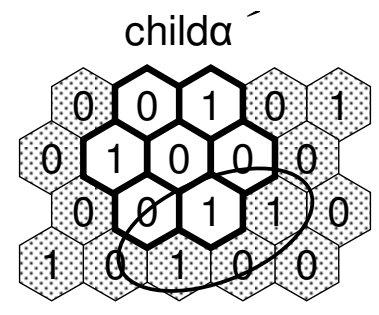

Figure 7. Interference after crossover
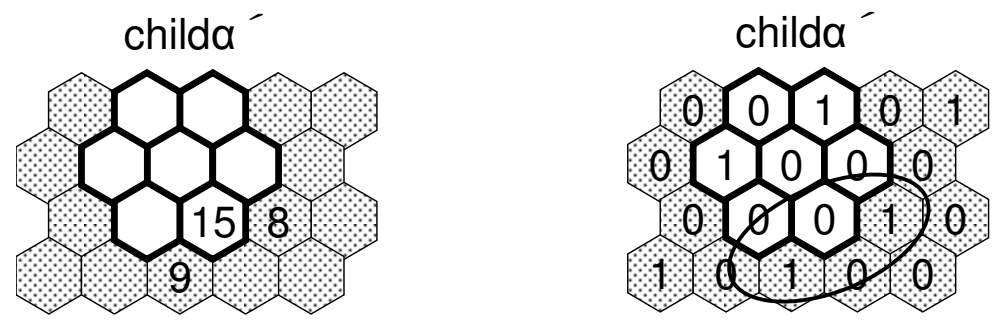

Figure 8. Operation after crossover (1)
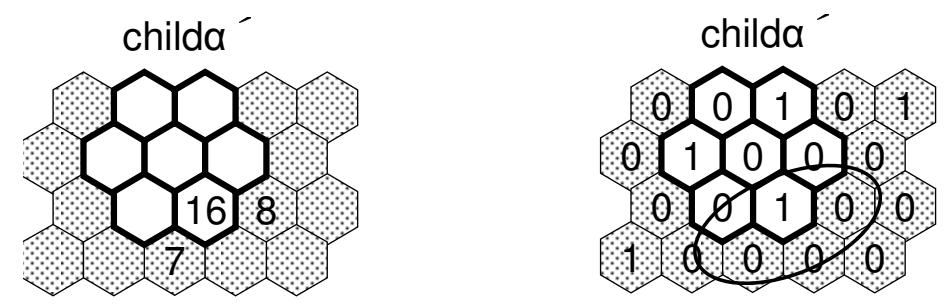

\subsection{Evaluation}

Figure 9. Operation after crossover (2)

At first, we define the load $Z_{i}$ of WiFi AP in cell $i$ as shown in Eq. (1), where $X_{i}$ and $Y_{i}$ denote the number of connected users in the cell and the number of available spectra of this AP, respectively.

$$
Z_{i}=\frac{X_{i}}{Y_{i}}
$$

Obviously, $Z_{i}$ should be updated in accordance with the changes of $X_{i}$. This update can be reflected by SM in real time.

Next, each individual is evaluated based on the sum of the load of WiFi APs that are spectrum assignment target. We define the fitness value $E$ as Eq. (2), where $I_{i}$ indicates the element of the list corresponding to cell $i$.

$$
E=\sum_{i=1}^{n} I_{i} Z_{i}
$$




\subsection{Selection}

After crossover, the number of individuals increases to $l+2 \mathrm{~m}$. To maintain the number of individuals, $2 m$ individuals are discarded by roulette selection. With the evaluated value $E_{j}$ of each individual, the survival probability $p_{j}$ of individual $j$ are defined by Eq. (3).

$$
p_{j}=\frac{E_{j}}{\sum_{j=1}^{l+2 m} E_{j}}
$$

$l$ individuals for survival are selected based on $p_{j}$ and the rest of individuals is discarded.

\subsection{Mutation}

If all individuals are identical, any new assignment patterns are never generated after crossover. To overcome this situation, mutation process is invoked to generate a different assignment pattern. We describe the mutation process with Figs. 10 and 11. First, a row is selected as a target of mutation. Next, the elements of all the cells in the target row and in its neighbour rows are temporarily changed to " 0 " as shown in Fig. 10. Note that, this process completely excludes interference in changing the elements of the cells of target rows. After that, the element of selected cell with a new WiFi AP differ from the one selected in Fig. 10 is changed to "1" as shown in Fig. 11. As a result, the assignment pattern of target row different from Fig. 10 is generated. Finally, in the cells of adjacent rows of the target row, the elements are changed to "1" to maximize the sum of the load after assignment.

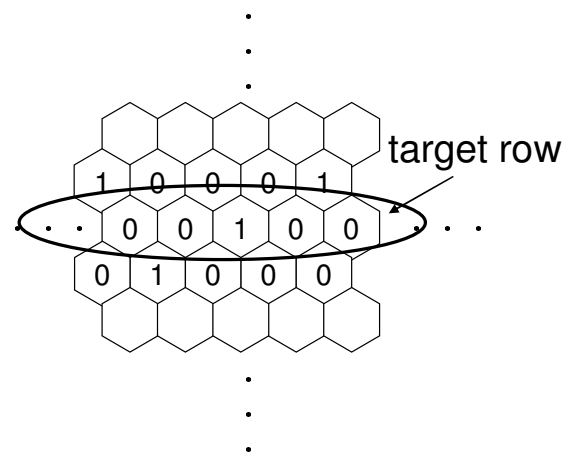

Figure 10. Mutation target

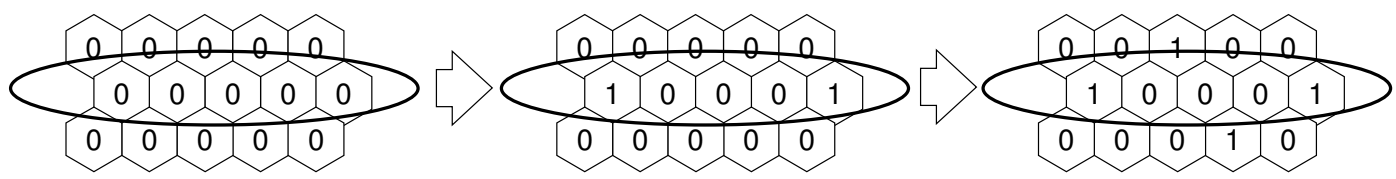

Figure 11. Mutation process

\subsection{Spectrum Assignment}

As described in section 3.1, GA continuously detects the solution in the proposed method and assigns spectrum based on the candidate individual at the time every constant time. 


\section{Performance Evaluation}

\subsection{Simulation Model}

In this section, we evaluate the performance of the proposed method to show its effectiveness by simulation experiments.

As a network model, we set one WiMAX BS including $10 \times 10=100$ cells where randomly selected 50 cells had WiFi AP.

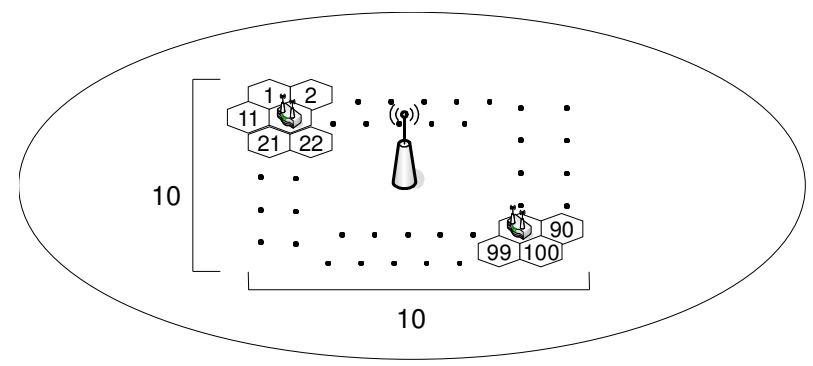

Figure 12. Simulation Model

The spectrum of the WiMAX BS was divided into several channels by the width of $10[\mathrm{MHz}]$ and each WiFi AP was allocated two channels. Each WiFi AP could use the one channel of $20[\mathrm{MHz}]$ except any channels assigned from WiMAX. In addition, WiMAX BS was assumed to provide 20[Mbps] per channel according to the evaluation in WiMAX Forum [19], and WiFi AP supported 17.5[Mbps] per channel according to our preliminary experiments using ns-2 [20]. The spectrum bandwidth of the WiMAX was set to $100[\mathrm{MHz}]$ and the interval time of spectrum assignment was set to $300[\mathrm{~s}]$. As a parameter setting of the proposed method, the number of individuals $l$ in the initial group was set to 10, the number of pairs of parents $m$ in crossover was set to 2 , the number of clusters of each individual was set to 20, and the number of clusters $k$ for crossover target was set to 7 . Furthermore, the number of the iteration of GA in the interval time of spectrum assignment was set to 1000 .

Supported traffic was BE traffic such as data downloading or web browsing. Users were supported to get a file whose size followed an exponential distribution with mean 10[MBytes]. When a new user arrived at a cell with WiFi AP, he/she used WiFi. Otherwise, he/she connected to WiMAX BS. In addition, users were staying in the arrived cell until the end of downloading. Calls occurred following a Poisson arrival process, and the arrival rate depended on the existence of WiFi AP. Generally speaking, because WiFi APs are set up in places where people gather (e.g., cafe and office), we set the arrival rate in a cell with WiFi AP to $\lambda$ and that in a cell without WiFi AP to $0.2 \lambda$.

As a compared method, we used the method that did not share any spectra (static). As a performance measure, we observed the time to complete the downloading over 10 experiments, where an experiment included 250,000 calls. 


\subsection{Simulation Results}

Fig. 13 shows the average download time as a function of arrival rate $\lambda$ for each method. At first, this result shows that two methods with spectrum sharing can greatly shorten the average download time compared with the method without it. This is because the capacity of integrated network was improved by allocating unused spectrum of WiMAX BS to WiFi AP.

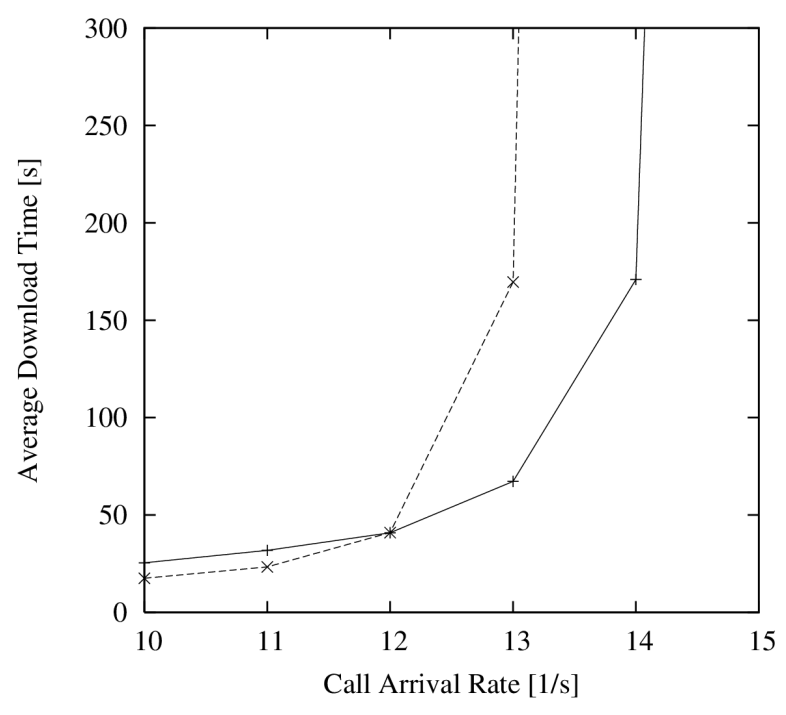

Figure 13. Simulation result

In addition, to evaluate the performance of GA, we used lp_solve (LP) [21] which is a mixed integer Linear Programming solver as a compared method. LP takes much time, so that it is not adequate for practical use. But, it can find quasi-optimal assignment pattern.

Fig. 14 shows that the download time by using GA and that by using LP are almost the same. In other words, the assignment pattern created by GA is almost optimal.

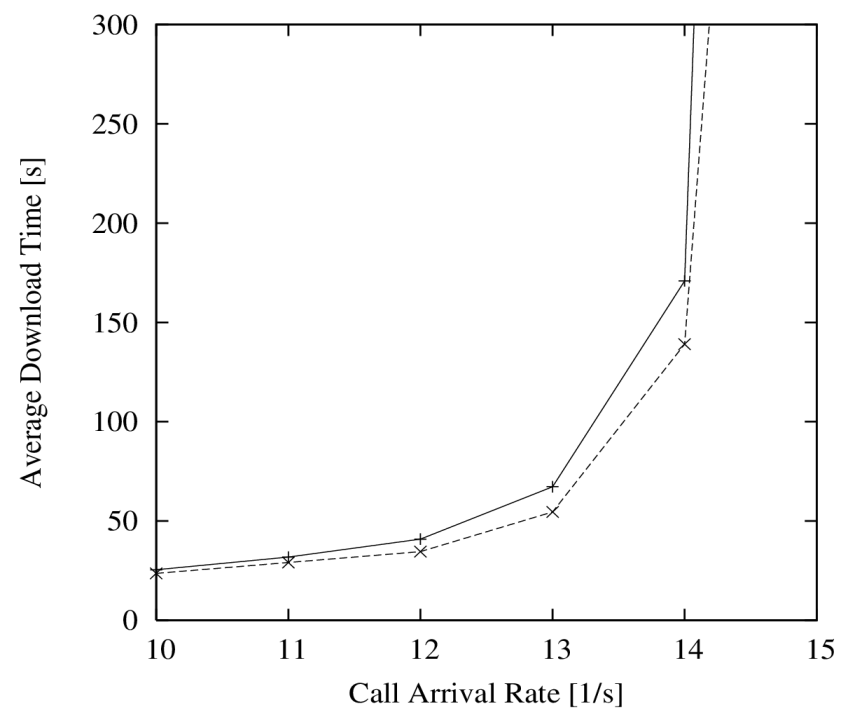

Figure 14. Optimality evaluation 
International Journal of Computer Networks \& Communications (IJCNC) Vol.6, No.5, September 2014

\section{Conclusions}

In this paper, we described the advances in wireless communication technologies and the lack of spectrum resources. Next, we have introduced integrated wireless network and DSA technology.

To use spectrum more efficiently in WiMAX/WiFi integrated network, we proposed the spectrum sharing method based on GA. In this method, to find WiFi APs to be assigned a spectrum from WiMAX BS, we used the sum of the load of WiFi AP as a fitness value and disallowed to assign the same spectrum to adjacent WiFi APs at the same time as a constraint. Finally, we showed that the proposed method could assign spectrum efficiently and improve user throughput by simulation experiments.

As a future work, we enhance the proposed method considered with handoff users.

\section{REFERENCES}

[1] K. Kinoshita, M. Nakagawa, K. Kawano, and K. Murakami, "A Fair and Efficient Spectrum Assignment for WiFi/WiMAX Integrated Networks," Proceedings of the 6th International Conference on Systems and Networks Communications (ICSNC 2011), October 2011.

[2] 3GPP, http://www.3gpp.org/.

[3] IEEE 802.11, http://grouper.ieee.org/groups/802/11/.

[4] “Air Interface for Fixed Broadband Wireless Access Systems," IEEE STD 802.16-2004, (Oct. 2004).

[5] “Air Interface for Fixed and Mobile Broadband Wireless Access Systems," IEEE P802.16e/D12\}, (Feb. 2005).

[6] S. Hanaoka, J. Yamamoto, M. Yano, "Platform for Load Balancing and Throughput Enhancement with Cognitive Radio," IEICE Transactions on Communications, vol. E91-B, no. 8, pp. 2501-2508, (Aug. 2008).

[7] L. Berlemann, C. Hoymann, G. R. Hiertz, S. Mangold, "Coexistence and Interworking of IEEE 802.16 and IEEE 802.11(e)," Vehicular Technology Conference, vol. 1, pp. 27-31, (May 2006).

[8] O. Khattab and O. Alani, "I Am 4 VHO: New Approach to Improve Seamless Vertical Handover in Heterogeneous Wireless Networks," International Journal of Computer Networks \& Communications (IJCNC) Vol.5, No.3, pp. 53-63, May 2013.

[9] J. Mitola III, "Cognitive Radio for Flexible Mobile Multimedia Communications," Mobile Networks and Applications, vol. 6, no. 5, (Sep. 2001).

[10] W. Krenik, A. Batra "Cognitive Radio Techniques for Wide Area Networks," Proceedings of the the 42nd annual Design Automation Conference, pp. 409-412, (Jun. 2005).

[11] M. Nekovee, "Dynamic spectrum access - concepts and future architectures," BT Technology Journal, vol. 24, no. 2, pp. 111-116, (Apr. 2006).

[12] Q. Zhao, B. M. Sadler, "A Survey of Dynamic Spectrum Access," IEEE Signal Processing Magazine, vol. 24, no. 3, pp. 79-89, (May 2007).

[13] E.Z. Tragos, S. Zeadally, A. G. Fragkiadakis, and V. A. Siris, "Sepctrum Assignment in Cognitive Radio Networks: A Comprehensive Survey," IEEE Communications Survey \& Tutorials, Vol. 15, No. 3, pp. 1108-1135 (2013).

[14] K. Kinoshita, Y. Kanamori, K. Kawano, and K. Murakami, "A Dynamic Spectrum Assignment Method for Call Blocking Probability Reduction in WiFi/WiMAX Integrated Networks," IEICE Transactions on Communications, vol. E94-B, no. 12, pp. 3498-3504, (Dec. 2011).

[15] J. Yoshino, I. Ohtomo, "Study on efficient channel assignment method using the genetic algorithm for mobile communication systems," Soft Computing - A Fusion of Foundations, Methodologies and Applications, vol. 9, no. 2, pp. 143-148, (Feb. 2005).

[16] C. Y. Ngo, V. O. K. Li, "Fixed Channel Assignment in Cellular Radio Networks Using a Modified Genetic Algorithm," IEEE Transactions on Vehicular Technology, vol. 47, no. 1, pp. 163-172, (Feb. 1998). 
[17] W. Song, W. Zhuang, and Y. Cheng, "Load Balancing for Cellular/WLAN Integrated Networks," IEEE Network, vol. 21, no. 1, pp. 27-33, (Jan.-Feb. 2007).

[18] D. Niyato, E. Hossain, "Integration of WiMAX and WiFi: Optimal Pricing for Bandwidth Sharing," IEEE Communications Magazine, vol. 45, no. 5, pp. 140-146, (May 2007).

[19] WiMAX Forum, http://www.wimaxforum.org/home/.

[20] ns-2, http://www.isi.edu/nsnam/ns/.

[21] lp_solve reference guide, http://lpsolve.sourceforge.net/5.5/.

\section{Authors}

Kazuhiko Kinoshita received the B.E., M.E. and Ph.D degrees in information systems engineering from Osaka University, Osaka, Japan, in 1996, 1997 and 2003, respectively. From April 1998 to March 2002, he was an Assistant Professor of the Department of Information Systems Engineering, Graduate School of Engineering, Osaka University. From April 2002 to March 2008, he was an Assistant Professor of the Department of Information Networking, Graduate School of Information Science

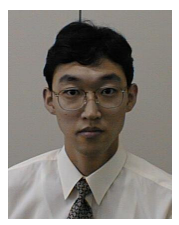
and Technology, Osaka University. Since April 2008, he has been an Associate Professor of the same University. His research interests include agent systems, mobile networks, and network architecture. Dr. Kinoshita is a member of IEEE.

Masashi Nakagawa received his bachelor's degree in engineering and master's degree in information science from Osaka University, Osaka, Japan, in 2009 and 2011, respectively. Since April 2011, he has been worked for the Ministry of Internal Affairs and Communications.

Keita Kawano received his B.E., M.E., and Ph.D. degrees from Osaka University, Osaka, Japan, in 2000, 2002, and 2004, respectively. Since October 2004, he has been an Assistant Professor of the Information Technology Center (at present, Center for Information Technology and Management), Okayama University, Okayama, Japan. His research interests include mobile communication networks and distributed systems. Dr. Kawano is a member of IEEE and IPSJ.

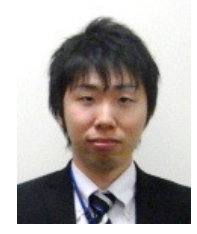

Koso Murakami received the B.E., M.E. and Ph.D degrees from Osaka University, Osaka, Japan in 1971, 1973 and 1991 respectively. From 1973 to 1995, he was with Fujitsu Laboratories Ltd., Kawasaki, Japan and engaged in research and development of digital switching systems, Asynchronous Transfer Mode switching systems and photonic switching technologies. From 1990 to 1995, he was a Manager of Communications Network Systems Laboratory of the same company and was responsible for research and development of broadband ISDN, Intelligent network,

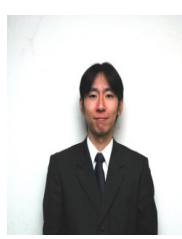

Telecommunications Management Network and personal communication networks. In April 1995, he joined Osaka University and was a Professor of Computation Center of the same University from 1995 to 1997. From 1998 to 2001, he was a Professor in the department of Information System Engineering of Osaka University. Since 2002, he has been a Professor of the Department of Information Networking in Graduate School of Information Science and Technology, newly established in Osaka University. From 2001 to 2004, he was concurrently Director of Collaborative Research Center of Advanced Science and Technology of the same University. From April 2004, he has served concurrently as Manager of Intellectual Property Office of Osaka University. His research interests extend to multimedia information networking architecture and photonic network. Prof. Murakami is a Fellow of IEICE and an IEEE Fellow. 